SEAH, TIEN HONG STANLEY, M.A. MAY 2019

PSYCHOLOGICAL SCIENCES

EMOTION DIFFERENTIATION AS A PROTECTIVE FACTOR AGAINST THE

BEHAVIORAL CONSEQUENCES OF RUMINATION: A CONCEPTUAL REPLICATION

AND EXTENSION IN THE CONTEXT OF SOCIAL ANXIETY (52 pp.)

Thesis Advisor: Karin G. Coifman

Rumination is thought to play a central role in affective disorders such as social anxiety disorder (SAD). Past research indicates that rumination tends to exacerbate negative emotions and increase the risk of engaging in maladaptive coping behaviors, e.g., avoiding social activities. However, little is known on how to effectively protect against the negative outcomes of rumination. Previously, Zaki, Coifman, Rafaeli, Berenson, and Downey (2013) found that negative emotion differentiation (NED) protected against rumination and non-suicidal self-injury in borderline personality disorder. Nevertheless, it is unclear whether this protective effect would extend to other populations and behaviors. Therefore, the present investigation sought to replicate and extend Zaki et al. (2013)'s findings in the context of SAD. In two studies, we examined if NED would moderate the relationship between rumination and the frequency of social avoidance. Study 1 involved 29 individuals who met criteria for SAD with or without major depressive disorder, while Study 2 involved 190 college students who responded to a selfreport measure of social anxiety. All participants completed a dispositional measure of rumination and an experience-sampling diary, which provided indices of NED and frequency of social avoidance. The results from both studies were unanimous: NED significantly moderated the relationship between rumination and social avoidance such that the positive association 
between rumination and social avoidance remained significant only for low NED. Overall, the findings provide a conceptual replication of Zaki et al. (2013) and further evidence for the protective effects of NED against the maladaptive consequences of rumination across populations. 


\title{
EMOTION DIFFERENTIATION AS A PROTECTIVE FACTOR AGAINST THE \\ BEHAVIORAL CONSEQUENCES OF RUMINATION: A CONCEPTUAL REPLICATION AND EXTENSION IN THE CONTEXT OF SOCIAL ANXIETY
}

\author{
A thesis submitted \\ To Kent State University in partial \\ Fulfillment of the requirements for the \\ Degree of Master of Arts
}

by

Tien Hong Stanley Seah

May, 2019

C Copyright

All rights reserved

Except for previously published materials 
Thesis written by

Tien Hong Stanley Seah

B.Soc.Sci., National University of Singapore, 2015

M.A., Kent State University, 2019

Approved by

Karin G. Coifman, Ph.D._, Advisor

Maria S. Zaragoza, Ph.D. , Chair, Department of Psychological Sciences

James L. Blank, Ph.D. , Dean, College of Arts and Sciences 


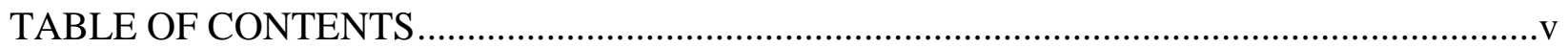

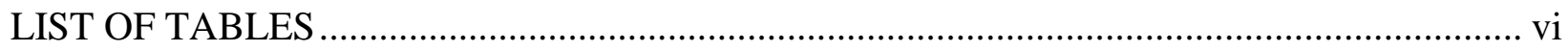

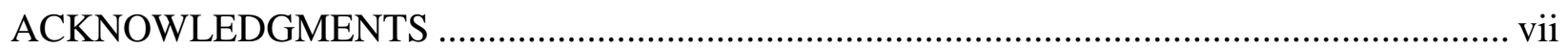

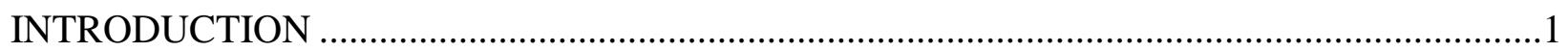

Rumination as a Risk Factor of Behavioral Dysregulation ............................................2

Emotion Differentiation as a Protective Factor ...............................................................

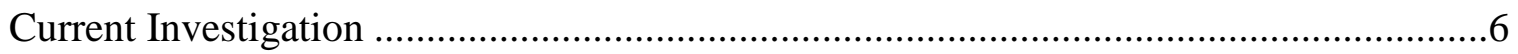

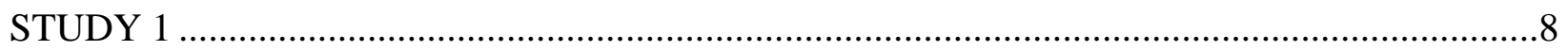

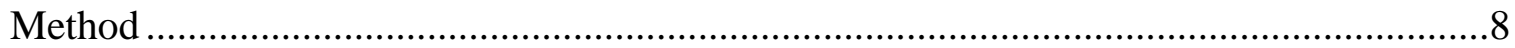

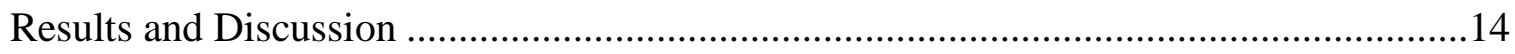

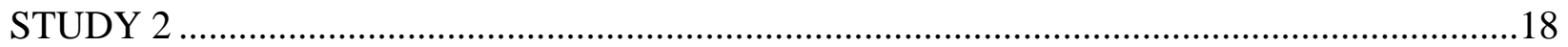

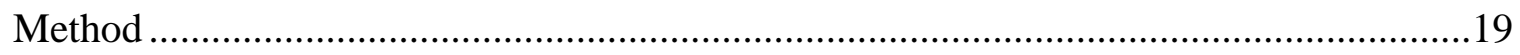

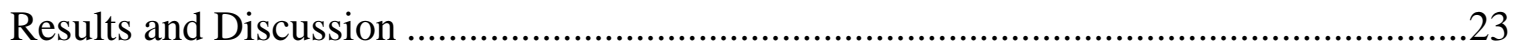

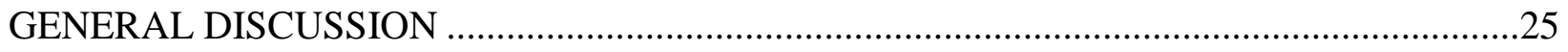

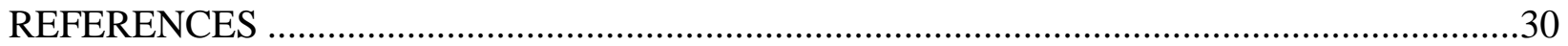




\section{LIST OF TABLES}

Table 1. Characteristics of Study 1 Participants $(n=54)$ .40

Table 2. Significant Two-way (Rumination by Negative Emotion Differentiation) Interaction in

Predicting Social Avoidance in the Clinical Group $(\mathrm{n}=29)$

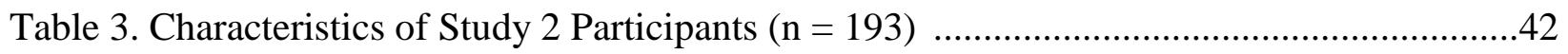

Table 4. Significant Two-way (Rumination by Negative Emotion Differentiation) Interaction in

Predicting Social Avoidance in the Sample $(n=190)$ 


\section{Acknowledgments}

The completion of this thesis marks another milestone in my academic journey and this was not possible without the assistance provided by the following individuals. First, I'd like to thank my advisor, Dr. Karin G. Coifman, for all the guidance, patience, and support while working with me on this project. Next, I'd like to thank my thesis committee members, Dr. Jeff Ciesla, Dr. Chris Was, and Dr. John Updegraff for their time and constructive feedback. Third, I am thankful for the support and laughter provided by my lab mates, Maria, Pallavi, and Chris, during the writing process. Finally, I am very grateful for all the kindness and encouragement provided by my friends (in the US and back home in Singapore) and family members throughout my time in graduate school. 


\section{Emotion Differentiation as a Protective Factor against the Behavioral Consequences of}

\section{Rumination: A Conceptual Replication and Extension in the Context of Social Anxiety}

Rumination is a maladaptive cognitive emotion regulation strategy (Aldao \& NolenHoeksema, 2010) often implicated in the development and maintenance of affective disorders such as depression and anxiety (Olatunji, Naragon-Gainey, \& Wolitzky-Taylor, 2013). It has been hypothesized to be a process of negative self-reference in response to negative emotions or events (Mennin \& Fresco, 2013) that typically results in increased levels of self-reported negative affect (Ciesla \& Roberts, 2007; Moberly \& Watkins, 2008; Nolen-Hoeksema, Stice, Wade, \& Bohon, 2007) and increases the risk of engaging in various maladaptive behaviors such as behavioral avoidance (Moulds, Kandris, Starr, \& Wong, 2007), substance use and binge eating (Nolen-Hoeksema et al., 2007), and non-suicidal self-injury (NSSI; Zaki, Coifman, Rafaeli, Berenson, \& Downey, 2013) across clinical and non-clinical populations. In the context of Social Anxiety Disorder (SAD), cognitive factors such as rumination are theorized to maintain the disorder by increasing the likelihood of engaging in maladaptive avoidance behaviors (e.g., avoid attending a social event) to reduce the anxiety of being in feared social situations (Hofmann, 2007). Despite playing a central role in affective disorders, little is known on how to effectively protect against the deleterious effects of rumination.

A potential protective factor may be the capacity to differentiate one's emotions into discrete categories, also known as emotion differentiation (ED). Individuals vary widely in their abilities to describe their emotional experiences. Some may describe their experiences broadly as feeling "good" or "bad", while others may do so with more specificity such as feeling "happy", 
"sad", or "anxious". Past research suggests that ED could mitigate the behavioral consequences following rumination. In one previous investigation, Zaki et al. (2013) found that negative ED (NED) mitigated the positive association between rumination and frequency of NSSI in borderline personality disorder. This finding builds upon previous studies that found significant inverse associations between NED and binge drinking (Kashdan, Ferssizidis, Collins, \& Muraven, 2010) and aggressive behaviors (Pond et al., 2012). However, it is unclear whether this protective effect would extend to other populations and maladaptive behaviors. Therefore, the present study sought to replicate the findings reported by Zaki et al. (2013) in the context of SAD. In two studies, as in Zaki et al. (2013), we predicted that negative emotion differentiation would moderate the relationship between rumination and the frequency of social avoidance (a maladaptive behavior characteristic of SAD) in a clinical sample of individuals with SAD and in a sample of socially anxious college students.

\section{Rumination as a Risk Factor of Behavioral Dysregulation}

Repetitive negative thinking styles such as rumination are theorized to be a transdiagnostic risk factor for various psychopathologies (Ehring \& Watkins, 2008) such as depression and anxiety disorders (Mclaughlin \& Nolen-Hoeksema, 2011) and post-traumatic stress disorder (Jenness et al., 2016). According to Nolen-Hoeksema (1991), rumination refers to the propensity to focus repeatedly about the causes, situational factors, and outcomes of one's emotional experience. Past research indicates that ruminating on one's negative emotional experience tends to increase the intensity of negative emotions (Donaldson \& Lam, 2004; Rusting \& Nolen-Hoeksema, 1998), and interacts with maladaptive cognitions leading to increases in self-reported negative affect (Ciesla \& Roberts, 2007). Further, this increase in negative affect may lead to increased levels of rumination, thereby resulting in a vicious 
feedback loop, termed an emotional cascade (Selby, Anestis, \& Joiner, 2008). Consequently, to terminate this repetitive cycle, individuals are thought to engage in behaviors (e.g., avoiding social situations in the case of social anxiety) that distract them from the cascade of maladaptive thoughts. Consistent with the emotional cascade hypothesis, previous research has found empirical support for the reciprocal relationship between rumination and negative affect (Moberly \& Watkins, 2008; Nolen-Hoeksema et al., 2007), sadness/depression and anxiety (see meta-analysis by Thomsen, 2006), and maladaptive behaviors, such as substance use and bingeeating in depression, substance abuse, and bulimia (Nolen-Hoeksema et al., 2007), NSSI in borderline personality disorder (Zaki et al., 2013), and social avoidance in college students (Moulds et al., 2007). Taken together, extant literature suggests that rumination is associated with a greater risk of engaging in maladaptive behaviors, particularly in response to negative emotional states, across various psychopathologies.

In the context of SAD, which is characterized by marked fears and avoidance of social and/or performance situations (American Psychiatric Association, 2013), ruminative thought processes are theorized to exacerbate and maintain the disorder (Hofmann, 2007). According to cognitive models of SAD (e.g., Heimberg, Brozovich, \& Rapee, 2010), maladaptive ruminative thought processes influence the severity of anxiety/fear of social situations as well as subsequent behaviors in response to these fears. These include anticipatory worry (thinking about an impending social situation, remembering previous failures, and forecasting poor performance or rejection), self-focused attention (directing attention towards internal self-relevant stimuli, e.g. physical appearance/response), and post-event review. As a result, the socially anxious individual avoids feared social situations in the future which could be extensive (e.g., not attending that event) or engages in a variety of safety behaviors (e.g., averting eye contact). Indeed, 
experimental evidence indicates that engaging in ruminative thought processes can lead to high levels of self-reported anxiety and development of greater maladaptive self-beliefs among highly socially anxious college students (Wong \& Moulds, 2009). Such maladaptive beliefs may in turn increase the propensity for individuals to avoid socially evaluative situations (Wong \& Moulds, 2011). This may work because avoidance, which typically involves engaging in behavioral strategies to avoid being in a feared situation, temporarily reduces anxiety. However, these strategies maintain the disorder by preventing opportunities to counter maladaptive cognitions and learn about the true threat value of the feared situation or stimuli (Clark \& Wells, 1995; Salkovskis, 1991). Further, the temporary relief experienced reinforces and perpetuates future avoidance behaviors (Mowrer, 1960). As such, avoidance is a critical target of treatment for SAD (Hofmann, 2007) and it is therefore pertinent to examine factors that could mitigate the occurrence of avoidance behaviors.

\section{Emotion Differentiation as a Protective Factor}

In contrast to rumination, some forms of attention to one's emotions can be adaptive and lead to beneficial outcomes. Various clinical theories and therapeutic interventions emphasize that the manner in which one observes negative emotional experience could influence the impact that these emotions have on subsequent affect, cognitions, and behaviors (cf. Hofmann \& Asmundson, 2008). For example, acceptance-based therapies teach individuals to observe and embrace their experiences non-judgmentally, so as to alter their experience of negative emotions. One way of attending to one's emotions adaptively that could interrupt the cycle of rumination and negative emotions is ED. ED involves recognizing, identifying, and labeling one's emotional experiences discretely, thereby making fine-grained distinctions between similarly valenced emotional states (Barrett, Gross, Christensen, \& Benvenuto, 2001). As discrete emotions offer 
information on how to cope with a situation (Schwarz \& Clore, 2003), the ability to differentiate one's emotions could be particularly advantageous in contexts involving intense negative emotions by promoting greater awareness, understanding, and regulation of emotional experiences (Barrett et al., 2001). This is in line with previous work demonstrating that labeling one's feelings reduced fear responses among spider phobics during an exposure exercise (Kircanski, Lieberman, \& Craske, 2012), self-reported distress among college students when viewing negative emotional pictures (Lieberman, Inagaki, Tabibnia, \& Crockett, 2011), and lower physiological reactivity during an exposure exercise for public speaking anxiety (Niles, Craske, Lieberman, \& Hur, 2015). Further, ED may serve as a pathway towards the adaptive regulation of negative emotions. For instance, ED has been associated with greater emotional clarity (Boden, Irons, Felder, Bujarski, \& Bonn-Miller, 2015), higher capacity to defuse from maladaptive thoughts (Plonsker, Biran, Zvielli, \& Bernstein, 2016), and lower emotional lability (Hill \& Updegraff, 2012).

Past research has found that individuals tend to vary widely in emotion differentiation capacities. Particularly, ED appears to be diminished among individuals with psychopathology involving affect and behavioral dysregulation such as social anxiety (Kashdan \& Farmer, 2014), depression (Demiralp et al., 2012), and borderline personality disorder (Suvak et al., 2011). As such, ED could play a critical role in emotional wellbeing by buffering against the use of maladaptive behavioral strategies to regulate negative emotions, such as substance use and binge-eating (Taylor, Bagby, \& Parker, 1997). Indeed, individuals who are more adept at differentiating their emotions (high differentiators) have reported being more able to identify and engage in adaptive strategies when regulating their emotions (Barrett et al., 2001). For instance, greater NED was associated with greater use of daily cognitive reappraisal among socially 
anxious college students (O’Toole, Jensen, Fentz, Zachariae, \& Hougaard, 2014). Further, high differentiators are less likely to consume excessive amounts of alcohol when stressed (Kashdan et al., 2010), demonstrate greater adherence to Thalassemia treatment (Coifman, Ross, Kleinart, \& Giardina, 2014), and less likely to relapse following substance use treatment (Anand, Chen, Lindquist, \& Daughters, 2017). Conversely, individuals who are less able to differentiate their emotions (low differentiators) are more susceptible to negative outcomes such as depression, neuroticism, and lower self-esteem (Erbas, Ceulemans, Pe, Koval, \& Kuppens, 2014), drinking excessive amounts of alcohol (Kashdan et al., 2010), greater likelihood of having angry outbursts (Pond et al., 2012), and higher frequency of NSSI (Zaki et al., 2013). Overall, these findings provide evidence that ED could protect against behavioral dysregulation, which is particularly relevant in contexts involving stress and intense negative emotions, where the need for emotion regulation may be the greatest.

\section{Current Investigation}

Presently, only one study has examined ED as a protective factor against the association between rumination and maladaptive behaviors. Zaki et al. (2013) found that NED moderated the positive association between rumination and frequency of NSSI behaviors in a sample of individuals with borderline personality disorder. Specifically, rumination predicted lower frequency of NSSI among individuals with moderate to high levels of NED but higher frequency of NSSI among low differentiators. Considering that rumination is argued to be a transdiagnostic risk factor of behavioral dysregulation, it remains pertinent to examine whether the protective effects of ED demonstrated in Zaki et al. (2013) would replicate and extend to other populations and behaviors. 
The present research sought to replicate Zaki et al. (2013) by examining whether NED protects against the behavioral consequences of rumination in the context of social anxiety (i.e., social avoidance). We investigated this research question in two different samples (clinical and non-clinical) using an experience-sampling approach as it provides a standard measure of NED and helps reduce the likelihood of retrospective biases commonly present in self-report research. The main hypothesis for the present investigation was guided by Zaki et al. (2013): we hypothesized that NED would moderate the association between rumination and social avoidance. First, in Study 1, we investigated this hypothesis in a sample of individuals with clinically diagnosed social anxiety. Specifically, we examined whether NED moderated the relationship between rumination and social avoidance, a feature characteristic of social anxiety. Like past studies (Suvak et al., 2011; Zaki et al., 2013), Study 1 included a healthy control group to demonstrate differences in rumination, NED, and social avoidance between socially anxious and healthy individuals to assist with the interpretation of the findings. In Study 2, we sought to examine if the same effect would extend further to a non-clinical sample comprised of socially anxious college students. To increase the reliability of our findings, we ensured that both studies had similar designs. These include having similar experience sampling protocols as well as using the same measures to assess rumination and social avoidance behaviors and urges. However, the studies differed on a few aspects. These include the duration of experience sampling (Study 1's was 14 days, while Study 2's was 10 days) and the assessment of social anxiety symptoms (a clinical diagnostic interview was administered in Study 1, while a self-report measure was used in Study 2). 


\section{Study 1}

\section{Method}

Participants. Participants who met criteria for a current DSM-IV diagnosis based on the DSM-IV-TR (American Psychiatric Association, 2000) of generalized social phobia (GSP) and/or major depressive disorder (MDD), or were determined to be healthy controls, were recruited as part of a larger study titled "Emotion, Attention, and Mood in Daily Life". Participants were recruited through printed flyers and postings at mental health clinics and public/community settings (e.g., libraries and gyms) in the community surrounding a large Midwestern university in the United States, as well as relevant list-servs and online websites. All participants were interviewed with the Structured Clinical Interview for DSM-IV Axis I Disorders (SCID-I; First, Gibbon, Spitzer, \& Williams, 1996), Structured Clinical Interview for DSM-IV-TR Axis II Personality Disorders (SCID-II; First, Gibbon, Spitzer, Williams, \& Benjamin, 1997) and supplemental modules from the Anxiety Disorders Interview Schedule Lifetime Version (ADIS IV-L; DiNardo, Brown \& Barlow, 1994).

In total, 45 individuals who met current diagnostic criteria for GSP and/or MDD were recruited for the larger study. For this clinical group, the inclusion criteria included meeting the diagnosis for social phobia (generalized subtype) with or without a major depressive episode based on the DSM-IV-TR (American Psychiatric Association, 2000). Exclusion criteria for the clinical group included meeting diagnostic criteria for bipolar disorder (I or II), borderline personality disorder, current psychosis, and current use of such medications as benzodiazepines, 
beta-blockers, tricyclic antidepressants, and antipsychotics. The exclusion of these medication classes was due to aspects of the larger study that involved examining autonomic nervous system activity, and these medication classes have been found to influence cardiovascular activity in ways that would interfere with the larger study's hypotheses.

To compare levels of rumination, social avoidance, and NED in the clinical group to those found in healthy individuals, 30 participants were recruited as a healthy control (HC) group after determining eligibility through the diagnostic interview. The inclusion criteria included an absence of any Axis-I disorder in the past year, absence of any personality disorder (less than two symptoms endorsed on any SCID-II scale), no use of psychiatric medications in the past year, global assessment of functioning (GAF) score on the SCID-I that was greater than 79, and no evidence of elevated social desirability (i.e., scores were not greater than 25 on the MarloweCrowne Social Desirability Scale; Crowne \& Marlowe, 1960).

A subsample of 60 individuals was identified from the larger sample for meeting inclusion criteria and completion of the experience sampling diary. Six participants who did not have a primary or co-occurring diagnosis of GSP were excluded due to the hypotheses of the current study. For the HC group, five participants dropped out of the study before the first study session while two participants did not provide any diary data. Therefore, the final sample comprised a total of 54 participants, with 31 in the clinical group, and 23 in the HC group. The mean (SD) age of the sample was 32.00 (12.59), where the clinical group $(M=34.97, S D=$ 13.38) was significantly older than the $\mathrm{HC}(M=28.00, S D=10.42), t(52)=-2.15, p=.036$. Descriptive information of both samples, including sex, race/ethnicity, and medication and treatment history are listed in Table 1. 
Procedure. Individuals who responded to the study flyers and online postings $(N=630)$ first participated in phone screenings conducted by trained research team members and were assessed for potential eligibility to participate in the larger study. These screenings adapted items from the SCID-I and the Interview Guide for Evaluating DSM-IV Psychiatric Disorders (Zimmerman, 1994) to assess for symptoms of MDD and GSP. Individuals $(n=158)$ who endorsed sufficient symptoms of MDD and GSP or did not endorse any symptoms were then invited to the laboratory for a detailed diagnostic assessment. Participants were compensated \$25 upon completion of the diagnostic assessment regardless of study eligibility.

Following the completion of the diagnostic interview, participants $(n=75)$ who met the eligibility criteria were assigned to their corresponding study groups (clinical or healthy) and began study participation. As part of the larger study, participants first completed a take-home questionnaire packet and subsequently returned to the laboratory to complete two separate study sessions. During these sessions, participants completed a series of cognitive and emotional tasks that were unrelated to the current investigation. At the first study session, all participants were trained by a research staff on the experience sampling diary portion of the study, which involved completing a 14-day electronic diary. Participants completed this portion of the study between the two laboratory sessions.

Participants were then compensated with $\$ 95$ for this portion of the study ( $\$ 15$ for completing the questionnaire packet, $\$ 30$ for the first study session, and $\$ 50$ for the experience sampling diary) and a $\$ 25$ bonus if they completed more than $90 \%$ of diary entries. All parts of the study were approved by the Kent State University Institutional Review Board prior to the start of any data collection. All participants provided written informed consent prior to the initial diagnostic interview and again if they were deemed eligible for the research study. 
Diagnostic interview. A structured diagnostic interview was administered to all participants. All interviews were conducted by clinical psychology doctoral students who were trained and supervised by a licensed clinical psychologist. Overall, the reliability for symptom and diagnostic level was reported to be good, average $\mathrm{K}>.90$. The diagnostic interview comprised of an assessment of current functioning, history of medical and psychiatric treatments, the SCID-1 (First et al, 1996), SCID II (for DSM-IV Axis II Personality Disorders; First et al., 1997), and the Generalized Anxiety Disorder and Social Phobia modules from the ADIS IV-L (DiNardo et al., 1994).

\section{Measures}

Rumination. The 5-item Brooding subscale of the Ruminative Responses Scale (RRS; Treynor, Gonzalez, \& Nolen-Hoeksema, 2003) was used to capture maladaptive rumination. It measures the frequency of engagement in ruminative thought processes when one feels depressed (e.g., "what am I doing to deserve this?”). Participants rated on a 4-point Likert scale ranging from 1 ("almost never") to 4 ("almost always"). An index of rumination was computed by obtaining the mean score across the ratings for all five items. The internal consistency of the Brooding subscale was high $(\alpha=.87)$.

Experience sampling diary. A 14-day electronic daily experience-sampling diary was used to measure the frequency of various behaviors and experience of positive and negative emotions. The experience-sampling data was collected using the Palm Centro personal desk assistant (PDA), programmed with the Purdue Momentary Assessment Tool (PMAT; www.cfs.purdue.edu/mfri/pages/PMAT/ Index .html). During the experience-sampling period, participants received five daily prompts at semi-random times over a 14-hour period daily for 14 days. Each diary prompt occurred at least 30 minutes after the previous diary, with a maximum 
gap of five hours in-between diary prompts. If needed, participants were able to delay or stop a diary prompt for up to 2 hours if their circumstances prevented them from answering the prompt (e.g., when driving a car). Therefore, participants could complete up to 70 diary entries. Four of the 54 participants were excluded from the present study due to insufficient diary data ${ }^{1}$. The remaining sample $(n=50)$ completed a mean $(\mathrm{SD})$ of $61.47(16.17)$ out of a possible 70 diary prompts (range $16-90$; compliance rate $=88 \%)^{2}$. There was no significant difference in the mean number of diaries completed by the clinical group $(M=64.55, S D=14.31)$ and the $\mathrm{HC}$ group $(M=57.10, S D=18.24), t(48)=-1.62, p=.112$.

Momentary self-reported emotions. During each diary prompt, participants were asked to rate their current emotional state using a 5-point Likert scale ranging from 1 ("not at all") to 5 (“extremely"). Participants responded to a series of emotion terms and rated the extent to which they presently felt each of six negative emotion words (fear, sadness, guilt, distress, anger, disgust), which comprised the negative affect scale. At each prompt, although unrelated to the hypotheses of the present investigation, participants also rated the extent to which they felt each of six positive emotion words (happiness, enjoyment, affection, satisfaction, amusement, relief). These negative and positive emotion words were selected to account for varying levels of activation across positive and negative valence dimensions of the circumplex model of affect (e.g., Russell, 1980). The reliability of the negative affect scale was computed at the betweenperson $\left(\mathrm{R}_{\mathrm{KF}}\right)$ and within-person $\left(\mathrm{K}_{\mathrm{C}}\right)$ levels based on the procedure recommended by Cranford et

\footnotetext{
${ }^{1}$ Two participants from each group were excluded. The criteria for exclusion due to insufficient diary entries was based on the recommendations by Bolger, Davis, \& Rafaeli (2003). This involved excluding participants who completed less than 13 diary entries, or less than 2 standard deviations from the average number of diaries responded across the sample. With the exception of age, there were no significant demographic or diagnostic differences between the excluded individuals and the final sample. As such, age was included as a covariate in subsequent analyses.

${ }^{2}$ Twelve participants completed more than 70 diary entries as they were late in returning the diary device. However, there were no significant differences in the primary outcome measures between individuals who completed more than 70 diaries compared to those who completed 70 diaries or less.
} 
al. (2006). The between-person reliability $\left(\mathrm{R}_{\mathrm{KF}}=.99\right)$ and within-person reliability $\left(\mathrm{R}_{\mathrm{C}}=.77\right)$ for this sample were good.

Mean negative affect. From the ratings of the negative emotion words, an index of mean negative affect (NA) was derived for each participant by calculating the mean score across the ratings of the six negative emotion words, across all diary prompts (Demiralp et al., 2012; Erbas et al., 2014).

Negative emotion differentiation. From the ratings of the negative emotion words, an index of NED was derived for each participant by computing the intra-class correlation coefficient (ICC) measuring absolute agreement (Tugade, Fredrickson, \& Barrett, 2004; Kashdan et al., 2010; Kashdan et al., 2014) across the ratings of the six negative emotion words across all diary prompts ${ }^{3}$. As a high ICC reflects low NED, they were subtracted from 1.0 to reverse the score such that higher values would indicate higher NED and lower values would indicate lower NED for ease of interpretation.

Momentary self-reported social avoidance. During each diary prompt, participants indicated if they engaged in social avoidance since the previous diary. They rated their engagement in social avoidance on a three-point scale ranging from "yes"; "no"; or "no but I thought about it a lot", which is deemed as an urge. An index of social avoidance was derived by first calculating an aggregate score consisting of either engaging in the behavior ("yes") or having the urge ("no but I thought about it a lot") to avoid social activities for each diary prompt. As in Zaki et al. (2013), to examine the rate at which individuals had urges to engage or engaged in social avoidance, a proportion score was created, which involved summing the aggregate

\footnotetext{
${ }^{3}$ Four individuals (all from the HC group) had negative ICCs. Following the procedures performed by Boden et al. (2013), these negative ICC scores were changed to 0 and included in subsequent analyses (cf. Cohen, Cohen, West, \& Aiken, 2003).
} 
score across all diaries for each participant and dividing it by the total number of diary prompts that the participant responded to (e.g., an overall aggregate score of 50 divided by 70 total diary prompts $=0.71$. . A square root transformation was applied as the distribution of scores was significantly positively skewed, $|\mathrm{z}|>3.29$ (since $50<\mathrm{n}<300$; Kim, 2013), which successfully removed the skew $(|z|=2.76)$. This was used as the dependent variable in subsequent analyses.

\section{Data Analytic Strategy}

First, means $(M)$ and standard deviations $(S D)$ of the brooding subscale, mean negative affect, negative emotion differentiation (NED), and social avoidance were obtained. Following which, a series of independent samples t-tests were executed to examine between-group differences on these outcomes. Then, as in Zaki et al. (2013), we tested the possible moderation of the association between rumination and social avoidance by NED using Ordinary Least Squares (OLS) regression with the Hayes PROCESS macro (Model 1; Hayes, 2013). This macro runs a series of Ordinary Least Squares (OLS) regressions with the centered product term representing the interaction of rumination by NED as a predictor of social avoidance. The estimated effects reported were unstandardized regression coefficients. Statistical significance was set at .05 .

\section{Results and Discussion}

Analyses involving both clinical and HC groups. First, group differences in rumination, mean NA, frequency of social avoidance, and NED were examined. As expected, the clinical group reported significantly higher levels of rumination ${ }^{4}(M=2.52, S D=0.85)$ than $\mathrm{HC}$ $(M=1.53, S D=0.46), t(45)=5.29, p<.001$, Cohen's $d=1.51,95 \%$ CI $[.88 ; 2.15]$. Across the

\footnotetext{
${ }^{4}$ Levene's test indicated unequal variances $(F=7.21, p=.010)$, so degrees of freedom were adjusted from 48 to 45 .
} 
diary period, the clinical group reported significantly higher levels of mean $\mathrm{NA}^{5}(M=1.58, S D=$ 0.50) compared to HC $(M=1.11, S D=0.09), t(31)=4.98, p<.001$, Cohen's $d=1.43,95 \% \mathrm{CI}$ $[.75 ; 2.08]$. Further, as anticipated, the clinical group reported significantly greater frequency of social avoidance $^{6}(M=0.41, S D=0.28)$ relative to $\mathrm{HC}(M=.07, S D=.10), t(38)=6.02, p<$ .001 , Cohen's $d=1.72,95 \%$ CI [1.04; 2.40], and significantly lower levels of $\operatorname{NED}^{7}(M=.37$, $S D=.19)$ compared to HC $(M=.54, S D=.32), t(30)=-2.20, p=.036$, Cohen's $d=-.63,95 \%$ CI $[-1.21 ;-.04]$.

We also obtained bivariate correlations between theoretically relevant variables of interest and the dependent variable across both groups. These include rumination, NED, mean NA, age, and sex. Notably, we found significant positive associations between rumination and social avoidance $(r=.40, p=.004)$, mean NA and social avoidance $(r=.64, p<.001)$, age and social avoidance $(r=.33, p=.018)$, and sex and social avoidance $(r=.35, p=.014)^{8}$. In contrast, NED was not significantly associated with social avoidance $(r=-.16, p=.278)$.

Primary analyses. Next, the primary hypotheses were examined in the clinical group. Within this group, we predicted that NED would moderate the positive association between dispositional levels of rumination and frequency of social avoidance. To examine NED as a moderator of the relationship between rumination and social avoidance, the Hayes PROCESS macro (Model 1; Hayes, 2013) was used. Rumination was entered as the independent variable

\footnotetext{
${ }^{5}$ Levene's test indicated unequal variances $(F=25.78, p<.001)$, so degrees of freedom were adjusted from 48 to 31.

${ }^{6}$ Levene's test indicated unequal variances $(F=13.73, p=.001)$, so degrees of freedom were adjusted from 48 to 38.

${ }^{7}$ Levene's test indicated unequal variances $(F=14.70, p<.001)$, so degrees of freedom were adjusted from 48 to 30 .

${ }^{8} \mathrm{We}$ also examined the same bivariate correlations for the clinical group but only found a significant positive association between mean NA and social avoidance $(r=.51, p=.005)$. There were no significant associations between rumination and social avoidance $(r=.10, p=.614)$, NED and social avoidance $(r=-.02, p=.911)$, age and social avoidance $(r=.36, p=.052)$, and sex and social avoidance $(r=.32, p=.092)$.
} 
(X), NED was entered as the moderator variable (M), and social avoidance (with square root transformation) was entered as the outcome variable (Y). Lastly, mean NA was entered as a covariate $^{9}$. The interaction product term was mean centered and standard errors were corrected for heteroscedasticity. The results of the moderation analysis are presented in Table 2 .

Overall, the regression model was significant, $R^{2}=.50, F(4,24)=7.10, p<.001$. Although there was no significant main effect of rumination on social avoidance $(B=.04, p=$ .575), as hypothesized, the results of the moderation analysis indicated that the interaction term between rumination and NED explained a significant increase in variance in social avoidance, $B$ $=-.78, \Delta R^{2}=.22, F(1,24)=7.90, p=.010$. Therefore, negative emotion differentiation significantly moderated the relationship between rumination and social avoidance.

To probe the effects of the interaction, predicted values were plotted for individuals $1 \mathrm{SD}$ above and below the mean of rumination and NED (refer to Figure 1). Follow-up tests of the simple slopes indicated that the association between rumination and social avoidance under low NED (1 SD below the mean) was significantly different from zero, $b=.18, p=.042$. However, the association between rumination and social avoidance under moderate $(b=.04, p=.575$. $)$ to high $(b=-.11, p=.198)$ NED was not significant. Therefore, for low differentiators, higher rumination predicted higher social avoidance. In contrast, this positive association between rumination and social avoidance was mitigated by moderate to high levels of NED, thereby protecting against the negative behavioral outcomes of rumination. Finally, we explored whether other potential covariates such as history of taking psychiatric medications and being in psychotherapy might impact the results but none of these variables significantly influenced our findings.

\footnotetext{
${ }^{9}$ Age and sex were also examined as covariates, but they did not have any significant effect on the results.
} 
The results of Study 1 were in line with our hypotheses and provide a conceptual replication of Zaki et al. (2013). First, group differences in rumination, mean NA, frequency of social avoidance, and NED between the clinical and HC groups were in the expected direction. Consistent with Kashdan and Farmer (2014), we found that NED was diminished in the clinical compared to the $\mathrm{HC}$ group. However, it was surprising that rumination did not have a significant direct effect on social avoidance in the clinical group. This may be due to limited variance as a result of elevated levels of rumination in the clinical group or small sample size. Next, NED significantly moderated the relationship between rumination and social avoidance. Specifically, the positive association between rumination and frequency of social avoidance was only significant for low but not moderate to high differentiators. These results remained even after controlling for such important covariates as mean levels of negative affect. A limitation of this study is its relatively small sample size. However, the sample size was comparable to similar experience sampling studies on social anxiety (Kashdan \& Collins, 2010; Kashdan \& Farmer, 2014) and sufficient to test the hypothesized interaction.

Overall, the results of Study 1 replicated and extended Zaki et al. (2013)'s findings in a sample of individuals with SAD. This provides further evidence for the protective effects of NED and builds upon previous work that found significant inverse associations between NED and maladaptive behaviors (Kashdan et al., 2010; Pond et al., 2012). Taken together, these findings indicate that NED could protect against the use of maladaptive behavioral strategies in response to negative emotional experiences. The convergence between Study 1 and Zaki et al. (2013)'s findings further suggests that ED could serve as a transdiagnostic protective factor against behavioral dysregulation. As such, we sought to replicate and extend the effects obtained in Study 1 in a non-clinical sample of socially anxious college students in Study 2. 


\section{Study 2}

Adolescence is a time period where peer and romantic relationships tend to become prominent in the adolescent's social life (Lasgaard, Goossens, Bramsen, Trillingsgaard, \& Elklit, 2011). These relationships are important because peer relations provide a sense of social integration while romantic partners may serve as reliable attachment figures (DiTommaso \& Spinner, 1997). However, adolescence is also a period of time where loneliness is prevalent and often associated with psychopathology (Heinrich \& Gullone, 2006). Particularly, peer-related and romantic loneliness were both found to be uniquely associated with social anxiety (Lasgaard et al., 2011). Additionally, loneliness has been associated with poorer social interaction quality characterized by less positive and more negative feelings during social interactions (Hawkley et al., 2003), low social competence, peer rejection and victimization, lack of high quality friendships, and low self-esteem (see review by Heinrich \& Gullone, 2006).

Despite experiencing loneliness, lonely individuals tend to withdraw or disengage rather than use adaptive strategies to cope with feelings of loneliness (Cacioppo et al., 2000). As a result, this maintains loneliness as the avoidance behaviors prevent them from engaging in activities that might reduce loneliness. For instance, in an experience sampling study by Kashdan, Barrios, Forsyth, and Steger (2006), the authors found that avoidance predicted daily levels of social anxiety and was inversely associated with positive daily events (such as participating in social activities) among college students. Given that loneliness is a problem often reported among college students (Ponzetti, 1990) and its unique associations with social anxiety, we were interested in examining whether the protective effects of ED obtained in Study 1 could 
extend to this population. Therefore, using a similar methodology as Study 1, we examined if NED might protect against rumination and social avoidance in a non-clinical sample of socially anxious college students.

\section{Method}

Participants. Participants were English-speaking college students (aged 18 and over; $N=$ 216) recruited through the research subject pool at Kent State University in the United States. From this initial sample of 216 individuals, a total of 23 individuals were excluded as they did not provide any diary data $(n=8)$, did not provide data on key study variables $(n=8)$, or failed accuracy checks $(n=7)^{10}$. Thus, the final sample comprised a total of 193 individuals. The mean (SD) age of the final sample was 20.07 (2.39). Descriptive information of the sample including sex, race/ethnicity, and undergraduate status are listed in Table 3.

Procedure. All participants were recruited as part of an online study titled "Emotion, Behaviors, and Social Context". Individuals who responded were directed to an online survey website where they were provided with the study information. All participants were given the opportunity to contact the study team if they had any questions or concerns about the study before providing informed consent. Following consent, all participants completed a series of questionnaires and were then provided with instructions to complete an online experiencesampling diary for 10 days. Upon completion of the online questionnaires and 10-day experience-sampling diary, participants were compensated with course credits. All parts of the study were approved by the Kent State University IRB prior to the start of data collection.

\footnotetext{
${ }^{10}$ Similar to other online studies (e.g., Gilman et al., 2017), accuracy checks were used to assess participants' engagement with the online content. These include having participants respond to two accuracy check questions: "Please type in the number 7" and "Please mark [the option] yes". Participants were excluded if the number of failed accuracy checks were more than 2 standard deviations above the sample's mean. With the exception of sex, there were no significant demographic differences between the excluded individuals and the final sample. Therefore, sex was included as a covariate in subsequent analyses.
} 


\section{Measures}

Social anxiety. The 24-item Liebowitz Social Anxiety Scale - Self Report version (LSAS; Heimberg et al., 1999; Fresco et al., 2001) was used to measure levels of social anxiety. The LSAS assesses fear and avoidance across 24 scenarios that tend to elicit social anxiety. Thirteen out of the 24 items relate to performance-based situations (e.g., writing while being observed, eating in public). The remaining 11 items relate to situations involving social interactions (e.g., being the center of attention, meeting strangers). For each item, individuals rated their levels of fear and avoidance of those situations in the past week using a 4-point Likert-type scale (ranging from 0 to 3 ). An index of social anxiety was obtained by summing the fear and avoidance ratings for all items. In this sample, the mean (SD) was 51.69 (25.46), which was below the traditional cut-off score of 55 or higher (indicating moderate levels of social anxiety) but was significantly higher compared to other college samples (e.g., $M=34.70, S D=$ 20.40; Russell \& Shaw, 2009). The internal consistency of the LSAS in this sample was high ( $\alpha$ $=.95)$.

Depression. The 20-item Center for Epidemiological Studies Depression Scale (CES-D; Radloff, 1977) was used to measure levels of depressive symptoms, with an emphasis on examining depressed mood. Items on the CES-D included components measuring depressed mood, feelings of guilt and worthlessness, helplessness, and hopelessness, psychomotor activity, changes in appetite, and sleep disruptions. For each item, participants rated how often they experienced the symptom in the past week from 0 ("rarely or none of the time") to 3 ("most or all of the time"). An index of depression was obtained by summing the ratings for all items. In this sample, the mean (SD) level of depression was 15.50 (10.07), which was below the traditional cut-off score of 16 or higher (indicating mild to moderate levels of depressive 
symptoms) but relatively higher compared to other college samples (e.g., $M=12.03, S D=8.98$;

Shields, Matt, \& Coifman, 2015). The internal consistency of the CES-D in this sample was $\operatorname{good}(\alpha=.78)$.

Rumination. As in Study 1, the 5-item Brooding subscale of the Ruminative Responses Scale (RRS; Treynor et al., 2003) was used to capture maladaptive rumination. In this sample, the mean (SD) was $2.13(0.82)$, which was relatively higher compared to other samples of college students (e.g., $M=1.72, S D=0.46$; Hoff \& Muehlenkamp, 2009). The internal consistency of the Brooding subscale was high $(\alpha=.86)$.

Experience sampling diary. A 10-day online experience-sampling diary was used to measure the frequency of various behaviors and experience of positive and negative emotions. The experience-sampling diary was administered through the secure online survey portal Qualtrics (www.qualtrics.com). Participants were able to respond to these online diaries using their smart phone and/or a computer at their convenience. During the experience-sampling period, participants received five daily e-mails that provided a link to the online diary to answer a series of questions. As in Study 1, participants were able to delay responding to a diary prompt if their circumstances prevented them from doing so. These prompts occurred at semi-random times, over a 12-hour period daily for 10 days. As in Study 1, during the daily 12-hour sampling period, each diary prompt occurred at least 30 minutes after the previous diary, with a maximum gap of five hours in-between diary prompts. As such, participants could complete up to 50 diary entries. Participants $(n=190)^{11}$ completed a mean (SD) of $31.64(12.43)$ out of a possible 50 diary prompts (range $4-50$; compliance rate $=63 \%$ ).

\footnotetext{
11 Three individuals were excluded from the analyses due to insufficient diary data. The criteria for exclusion was similar to Study 1 based on recommendations by Bolger et al. (2003). This involved excluding participants who completed less than 4 diary entries, or less than 2 standard deviations from the average number of diaries responded
} 
Momentary self-reported emotions. As in Study 1, during each diary prompt, participants were asked to rate their current emotional state by responding to the same set of six positive and six negative emotion words. The between-person reliability $\left(\mathrm{R}_{\mathrm{KF}}=.97\right)$ and within-person reliability $\left(\mathrm{R}_{\mathrm{C}}=.77\right)$ of the negative affect scale for this sample were good.

Mean negative affect. As in Study 1, an index of mean negative affect (NA) was derived for each participant by calculating the mean score across the ratings of the six negative emotion words, across all diary prompts. In this sample, the mean (SD) negative affect reported was 1.53 (0.59).

Negative emotion differentiation. As in Study 1, an index of NED was derived for each participant by computing the average intra-class correlation coefficient (ICC) measuring absolute agreement across the ratings of the six negative emotion words across all diary prompts ${ }^{12}$. These scores were then subtracted from 1.0 to reverse the score such that higher values would indicate higher NED and lower values would indicate lower NED for ease of interpretation. The mean (SD) levels of NED reported in this sample was .46 (.29), which is relatively lower compared to other samples of college students (e.g., $M=.59, S D=.14$; Kashdan et al., 2010)

Momentary self-reported social avoidance. As in Study 1, during each diary prompt, participants indicated if they engaged in social avoidance since the previous diary. They rated their engagement in social avoidance on a three-point scale ranging from "yes"; "no"; or "no but I thought about it a lot" (urge). As in Study 1 and Zaki et al. (2013), an index of social avoidance was derived by calculating a proportion score to examine the rate at which individuals had urges to engage or engaged in social avoidance. The mean (SD) frequency of social avoidance across

across the sample. There were no significant demographic differences between the excluded individuals and the final sample.

${ }^{12}$ Sixteen individuals reported negative ICCs. Following the procedures used in Study 1, these negative ICC scores were changed to 0 and included in subsequent analyses. 
all diaries in this sample was 0.05 (0.08). However, a square root transformation was applied as the distribution of scores was found to be significantly positively skewed, $|z|>3.29$ (since $50<n$ <300; Kim, 2013). Despite applying the square root transformation, the distribution remained significantly positively skewed. As such, the Winsor procedure (Guttman, 1973) was used to truncate the distribution by substituting the highest value in the distribution with the next highest value. This was performed eight times, until the positive skew was removed $(|z|=3.13)^{13}$.

\section{Data Analytic Strategy}

As in Study 1, the moderation of the association between rumination and social avoidance by NED was tested using Ordinary Least Squares (OLS) regression with the Hayes PROCESS macro (Model 1; Hayes, 2013). Again, the estimated effects reported were unstandardized regression coefficients and statistical significance was set at .05.

\section{Results and Discussion}

As in Study 1, we first examined bivariate correlations between theoretically relevant variables of interest and the dependent variable to observe the data for potential covariates. Social avoidance was only significantly correlated with rumination $(r=.28, p<.001)$ and mean NA $(r=.30, p<.001)$. As such, mean NA was subsequently included as a covariate in the regression model. The results of the moderation analysis are presented in Table 4. Overall, the regression model was significant, $R^{2}=.18, F(4,185)=8.13, p<.001$. Consistent with Study 1 , although there was no significant main effect of rumination on social avoidance $(B=.02, p=$ $.153)$, as hypothesized, the results of the moderation analysis indicated that the interaction term between rumination and NED explained a significant increase in variance in social avoidance, $B$

\footnotetext{
${ }^{13}$ To examine if the truncation procedure influenced the significance of the findings, the same analyses were performed without truncating the distribution and the results obtained were the same.
} 
$=-.14, \Delta R^{2}=.03, F(1,185)=6.94, p=.009$. Therefore, as in Study 1, NED significantly

moderated the relationship between rumination and social avoidance ${ }^{14}$.

To probe the effects of the interaction, predicted values were plotted for individuals $1 \mathrm{SD}$ above and below the mean of rumination and NED (refer to Figure 2). Follow-up tests of the simple slopes indicated that the association between rumination and social avoidance under low NED (1 SD below the mean) was significantly different from zero, $b=.06, p=.003$. However, the association between rumination and social avoidance under moderate $(b=.02, p=.153)$ to high $(b=-.02, p=.428)$ NED was not significant. Therefore, for low differentiators, higher rumination predicted higher social avoidance. In contrast, this positive association between rumination and social avoidance was mitigated by moderate to high levels of NED, thereby protecting against the negative behavioral outcomes of rumination.

Replicating Study 1's findings, NED significantly moderated the relationship between rumination and frequency of social avoidance. As in Study 1, the positive association between rumination and frequency of social avoidance was only significant for low but not moderate to high differentiators. These results remained even after controlling for mean NA. These replications show that the effects found in Study 1 are reliable and extend to non-clinically socially anxious college students. This suggests that NED serves as a protective factor even for individuals with subthreshold psychopathological symptoms. However, replication of these findings in non-clinical community samples in future studies would further strengthen these findings.

\footnotetext{
${ }^{14}$ Sex and depression were also examined as additional covariates in the moderation analysis but did not have any meaningful effects on the results.
} 


\section{General Discussion}

Given the central role of rumination in various psychopathologies, particularly affective disorders such as $\mathrm{SAD}$, it is important to examine factors that might protect against its negative consequences. In two studies, we explored whether ED could serve as a protective factor against the behavioral outcomes (social avoidance) of rumination in a sample of individuals with clinically diagnosed SAD as well as a non-clinical sample of socially anxious college students. Overall, the results from both studies unanimously support our central hypothesis: NED significantly moderated the relationship between rumination and frequency of social avoidance such that the positive association between rumination and social avoidance was only significant for low but not moderate to high differentiators. This consistent finding obtained from two different samples demonstrate that the ability to discriminate among negative emotions is protective against the maladaptive outcomes of rumination. Further, our results replicate and expand upon previous findings reported by Zaki et al. (2013). This suggests that ED could serve as a transdiagnostic protective factor against rumination and behavioral dysregulation.

Building upon cognitive models of SAD (see Hofmann, 2007), the present investigation revealed that the impact of maladaptive cognitive processes such as rumination on avoidance behaviors is influenced by other emotional processes like emotion differentiation. To our knowledge, this is the first study that examined rumination together with emotion differentiation in predicting avoidance behaviors in the context of social anxiety. Like Zaki et al. (2013), our findings also build on the emotional cascade model by Selby et al. (2008) suggesting that the capacity to identify, discriminate, and label one's emotions during a negative emotional 
experience may help reduce the likelihood of engaging in maladaptive behaviors to terminate the repetitive ruminative cycle. This ties in with previous work demonstrating the emotion regulatory benefits of using words to label one's feelings (Lieberman et al., 2011; Kircanski et al., 2012; Niles et al., 2015), which is argued to work through dampening amygdala activation via the prefrontal cortex thereby reducing physiological arousal (Lieberman et al., 2007). For example, individuals who labeled their emotional experiences during a public speaking exposure exercise demonstrated greater decreases in physiological reactivity compared to those who did not use any labels (Niles et al., 2015). Further, these reductions in physiological reactivity positively correlated with the number of labels used during the exposure exercise. Taken together, these findings suggest that labeling one's emotional experience discretely helps dampen its intensity. This could be particularly useful in contexts involving intense negative emotions, such as in an emotional cascade, where the ability to label one's emotional experience may decrease its intensity and prevent the use of maladaptive strategies to cope with negative emotions. Nevertheless, this hypothesis warrants further investigation. Future studies should consider exploring the relationship between emotion labeling and other maladaptive behaviors, particularly in high risk populations (e.g., suicide risk in the context of depression).

Our findings also contribute to extant literature suggesting that ED could function as a general protective factor against various maladaptive behavioral outcomes in emotionally at-risk individuals (e.g., binge drinking, aggression, NSSI). In spite of these positive findings as well as the recent surge of research on $\mathrm{ED}$, the precise mechanisms for its salutary effects are not yet well understood (Kashdan, Barrett, \& McKnight, 2015). One possibility is that using emotion words or labels to categorize emotional experiences generates a conceptualization of the sensations linked to that experience, which then provides specific predictions for (adaptive) 
action in that context (Kashdan et al., 2015). For example, "I feel sad, therefore I should talk to someone about it to feel better." Another possibility could be that using labels to describe one's emotional experiences reduces physiological reactivity, which then enables one to defuse from the experience and adopt the psychological distance necessary to adaptively process and reflect on emotional experiences. By taking a distanced perspective, it allows for a broader focus and reappraisal of experiences thereby reducing rumination and distress (Kross, Ayduk, \& Mischel, 2005; Kross \& Ayduk, 2011; Kross, Gard, Deldin, Clifton, \& Ayduk, 2012). This ties in with acceptance-based interventions where individuals are taught the technique of defusion by learning to observe, identify, and label their experiences (Hayes, Strosahl, \& Wilson, 1999). However, beyond these preliminary hypotheses, there remain numerous unanswered questions: Does the specificity or accuracy of the emotion label matter? How do other cognitive processes such as working memory impact ED? Future research should aim to uncover the basic processes underlying ED, which could then be used to inform psychological interventions.

The results of the present investigation need to be interpreted in light of the following limitations. First, we did not include a clinical control group in Study 1. Given that rumination also plays a central role in other affective disorders (such as depression and post-traumatic stress disorder), future studies could compare ED in these populations to further understand the relationship of ED and maladaptive behaviors in affective disease. Second, the use of experience sampling methodology likely inflicted burden on participants and may have given rise to demand characteristics. For instance, requiring participants to monitor themselves daily could influence the reported rate of social avoidance behaviors and urges (Bolger et al., 2003). Lastly, the present study only examined how trait levels of rumination influenced the frequency of social avoidance. Considering that cognitive, affective, and behavioral processes in SAD are context-specific (e.g., 
before, during, or after feared social situations), it will be useful to examine how ED might moderate the link between momentary rumination on subsequent behaviors. Future investigations are currently underway.

Despite these limitations, the current investigation also had several strengths. First, except for the experience sampling duration, the measures used in both studies were equivalent. This equivalence in methodology increases the reliability and validity of our results, which enables us to derive more conclusive interpretations of the findings. Further, the use of experiencing sampling methods reduces retrospective biases that are often problematic in selfreport research and increases the ecological validity of our findings. Next, we used both a clinical and non-clinical sample of socially anxious individuals and were able to replicate the protective effects of ED in both studies. This demonstrates the robustness of our findings and provides further support for ED as a protective factor across different populations.

Overall, the findings reported in the present investigation contribute to an emerging literature indicating that ED provides resiliency against various maladaptive behaviors. Further, consistent with Zaki et al. (2013), our findings provide support for the protective effect of ED against rumination demonstrating that ED buffers against social avoidance among ruminating individuals with social anxiety. The results also provide empirical support for various cognitive theories and treatment models of SAD where the ability to identify and label emotions, particularly in stressful contexts, are associated with more adaptive outcomes. These findings have important clinical implications for understanding and treating SAD as well as other affective disorders involving behavioral dysregulation. Nevertheless, more research is needed to understand the basic processes underlying ED and to elucidate the mechanisms through which 
ED exerts its beneficial effects. In so doing, it is hoped that the knowledge gleaned would be used to inform and improve therapeutic treatments for various affective disorders. 


\section{References}

Aldao, A., \& Nolen-Hoeksema, S. (2010). Specificity of cognitive emotion regulation strategies: A transdiagnostic examination. Behaviour Research and Therapy, 48(10), 974-983.

American Psychiatric Association. (2000). Diagnostic and statistical manual of mental disorders (revised 4th ed.). Washington, DC: Author.

American Psychiatric Association. (2013). Diagnostic and statistical manual of mental disorders (5 $5^{\text {th }}$ edition). Arlington, VA: American Psychiatric Publishing.

Anand, D., Chen, Y., Lindquist, K. A., \& Daughters, S. B. (2017). Emotion differentiation predicts likelihood of initial lapse following substance use treatment. Drug and Alcohol Dependence, 180, 439-444.

Barrett, L. F., Gross, J., Christensen, T. C., \& Benvenuto, M. (2001). Knowing what you're feeling and knowing what to do about it: Mapping the relation between emotion differentiation and emotion regulation. Cognition \& Emotion, 15(6), 713-724.

Boden, M. T., Irons, J. G., Feldner, M. T., Bujarski, S., \& Bonn-Miller, M. O. (2015). An investigation of relations among quality of life and individual facets of emotional awareness and mindfulness. Mindfulness, 6(4), 700-707.

Boden, M. T., Thompson, R. J., Dizén, M., Berenbaum, H., \& Baker, J. P. (2013). Are emotional clarity and emotion differentiation related?. Cognition \& Emotion, 27(6), 961-978.

Bolger, N., Davis, A., \& Rafaeli, E. (2003). Diary methods: Capturing life as it is lived. Annual Review of Psychology, 54(1), 579-616. 
Brown, T. A., Barlow, D. H., \& DiNardo, P. A. (1994). Anxiety Disorders Interview Schedule for DSM-IV (ADIS-IV): Client Interview Schedule. Graywind Publications Incorporated.

Cacioppo, J. T., Ernst, J. M., Burleson, M. H., McClintock, M. K., Malarkey, W. B., Hawkley, L. C., ... \& Spiegel, D. (2000). Lonely traits and concomitant physiological processes: The MacArthur social neuroscience studies. International Journal of Psychophysiology, 35(23), 143-154.

Ciesla, J. A., \& Roberts, J. E. (2007). Rumination, negative cognition, and their interactive effects on depressed mood. Emotion, 7(3), 555.

Clark, D. M., \& Wells, A. (1995). A cognitive model of social phobia. In M. Liebowitz \& R. G. Heimberg (Eds.), Social phobia: Diagnosis, assessment, and treatment (pp. 69-93). New York: Guilford Press.

Cohen, J., Cohen, P., West, S. G., \& Aiken, L. S. (2003). Applied multiple correlation/regression analysis for the behavioral sciences. UK: Taylor \& Francis.

Coifman, K. G., Ross, G. S., Kleinert, D., \& Giardina, P. (2014). Negative affect differentiation and adherence during treatment for thalassemia. International Journal of Behavioral Medicine, 21(1), 160-168.

Cranford, J. A., Shrout, P. E., Iida, M., Rafaeli, E., Yip, T., \& Bolger, N. (2006). A procedure for evaluating sensitivity to within-person change: Can mood measures in diary studies detect change reliably?. Personality and Social Psychology Bulletin, 32(7), 917-929.

Crowne, D. P., \& Marlowe, D. (1960). A new scale of social desirability independent of psychopathology. Journal of Consulting Psychology, 24(4), 349-354. 
Demiralp, E., Thompson, R. J., Mata, J., Jaeggi, S. M., Buschkuehl, M., Barrett, L. F., ... \& Gotlib, I. H. (2012). Feeling blue or turquoise? Emotional differentiation in major depressive disorder. Psychological Science, 23(11), 1410-1416.

DiTommaso, E., \& Spinner, B. (1997). Social and emotional loneliness: A re-examination of Weiss' typology of loneliness. Personality and Individual Differences, 22(3), 417-427.

Donaldson, C., \& Lam, D. (2004). Rumination, mood and social problem-solving in major depression. Psychological Medicine, 34(7), 1309-1318.

Ehring, T., \& Watkins, E. R. (2008). Repetitive negative thinking as a transdiagnostic process. International Journal of Cognitive Therapy, 1(3), 192-205.

Erbas, Y., Ceulemans, E., Lee Pe, M., Koval, P., \& Kuppens, P. (2014). Negative emotion differentiation: Its personality and well-being correlates and a comparison of different assessment methods. Cognition and Emotion, 28(7), 1196-1213.

First, M. B., Gibbon, M., Spitzer, R. L., \& Williams, J. B. (1996). User's guide for the structured clinical interview for DSM-IV axis I Disorders-Research version. New York: Biometrics Research Department, New York State Psychiatric Institute.

Fresco, D. M., Coles, M. E., Heimberg, R. G., Liebowitz, M. R., Hami, S., Stein, M. B., \& Goetz, D. (2001). The Liebowitz Social Anxiety Scale: a comparison of the psychometric properties of self-report and clinician-administered formats. Psychological Medicine, 31(6), 1025-1035.

Gibbon, M., Spitzer, R. L., Williams, J. B., Benjamin, L. S., \& First, M. B. (1997). Structured clinical interview for DSM-IV axis II personality disorders (SCID-II). American Psychiatric Publishing. 
Gilman, T. L., Shaheen, R., Nylocks, K. M., Halachoff, D., Chapman, J., Flynn, J. J., ... \& Coifman, K. G. (2017). A film set for the elicitation of emotion in research: A comprehensive catalog derived from four decades of investigation. Behavior Research Methods, 49(6), 2061-2082.

Guttman. (1973). Premium and protection of several procedures for dealing with outliers when sample sizes are moderate and large. Technometrics, 15, 385-404.

Hawkley, L. C., Burleson, M. H., Berntson, G. G., \& Cacioppo, J. T. (2003). Loneliness in everyday life: Cardiovascular activity, psychosocial context, and health behaviors. Journal of Personality and Social Psychology, 85(1), 105-120.

Hayes, A. F. (2013). Model templates for PROCESS for SPSS and SAS. Retrieved August 8, 2018.

Hayes, S. C., Strosahl, K. D., \& Wilson, K. G. (1999). Acceptance and commitment therapy. New York: Guilford Press.

Heimberg, R. G., Brozovich, F. A., \& Rapee, R. M. (2010). A cognitive behavioral model of social anxiety disorder: Update and extension. In Social Anxiety (Second Edition) (pp. 395-422).

Heimberg, R. G., Horner, K. J., Juster, H. R., Safren, S. A., Brown, E. J., Schneier, F. R., \& Liebowitz, M. R. (1999). Psychometric properties of the Liebowitz social anxiety scale. Psychological Medicine, 29(1), 199-212.

Heinrich, L. M., \& Gullone, E. (2006). The clinical significance of loneliness: A literature review. Clinical Psychology Review, 26(6), 695-718.

Hill, C. L., \& Updegraff, J. A. (2012). Mindfulness and its relationship to emotional regulation. Emotion, 12(1), 81-90. 
Hoff, E. R., \& Muehlenkamp, J. J. (2009). Nonsuicidal self-injury in college students: The role of perfectionism and rumination. Suicide and Life-Threatening Behavior, 39(6), 576-587.

Hofmann, S. G. (2007). Cognitive factors that maintain social anxiety disorder: A comprehensive model and its treatment implications. Cognitive Behaviour Therapy, 36(4), 193-209.

Hofmann, S. G., \& Asmundson, G. J. (2008). Acceptance and mindfulness-based therapy: New wave or old hat?. Clinical Psychology Review, 28(1), 1-16.

Jenness, J. L., Jager-Hyman, S., Heleniak, C., Beck, A. T., Sheridan, M. A., \& McLaughlin, K. A. (2016). Catastrophizing, rumination, and reappraisal prospectively predict adolescent PTSD symptom onset following a terrorist attack. Depression and Anxiety, 33(11), 10391047.

Kashdan, T. B., Barrett, L. F., \& McKnight, P. E. (2015). Unpacking emotion differentiation: Transforming unpleasant experience by perceiving distinctions in negativity. Current Directions in Psychological Science, 24(1), 10-16.

Kashdan, T. B., Barrios, V., Forsyth, J. P., \& Steger, M. F. (2006). Experiential avoidance as a generalized psychological vulnerability: Comparisons with coping and emotion regulation strategies. Behaviour Research and Therapy, 44(9), 1301-1320.

Kashdan, T. B., \& Collins, R. L. (2010). Social anxiety and the experience of positive emotion and anger in everyday life: An ecological momentary assessment approach. Anxiety, Stress, \& Coping, 23(3), 259-272.

Kashdan, T. B., \& Farmer, A. S. (2014). Differentiating emotions across contexts: Comparing adults with and without social anxiety disorder using random, social interaction, and daily experience sampling. Emotion, 14(3), 629-638. 
Kashdan, T. B., Ferssizidis, P., Collins, R. L., \& Muraven, M. (2010). Emotion differentiation as resilience against excessive alcohol use: An ecological momentary assessment in underage social drinkers. Psychological Science, 21(9), 1341-1347.

Kim, H. Y. (2013). Statistical notes for clinical researchers: Assessing normal distribution (2) using skewness and kurtosis. Restorative Dentistry \& Endodontics, 38(1), 52-54.

Kircanski, K., Lieberman, M. D., \& Craske, M. G. (2012). Feelings into words: contributions of language to exposure therapy. Psychological Science, 23(10), 1086-1091.

Kross, E., \& Ayduk, O. (2011). Making meaning out of negative experiences by selfdistancing. Current Directions in Psychological Science, 20(3), 187-191.

Kross, E., Ayduk, O., \& Mischel, W. (2005). When asking “why” does not hurt distinguishing rumination from reflective processing of negative emotions. Psychological Science, 16(9), 709-715.

Kross, E., Gard, D., Deldin, P., Clifton, J., \& Ayduk, O. (2012). “Asking why” from a distance: Its cognitive and emotional consequences for people with major depressive disorder. Journal of Abnormal Psychology, 121(3), 559.

Lasgaard, M., Goossens, L., Bramsen, R. H., Trillingsgaard, T., \& Elklit, A. (2011). Different sources of loneliness are associated with different forms of psychopathology in adolescence. Journal of Research in Personality, 45(2), 233-237.

Lieberman, M. D., Eisenberger, N. I., Crockett, M. J., Tom, S. M., Pfeifer, J. H., \& Way, B. M. (2007). Putting feelings into words. Psychological Science, 18(5), 421-428.

Lieberman, M. D., Inagaki, T. K., Tabibnia, G., \& Crockett, M. J. (2011). Subjective responses to emotional stimuli during labeling, reappraisal, and distraction. Emotion, 11(3), 468480. 
McLaughlin, K. A., \& Nolen-Hoeksema, S. (2011). Rumination as a transdiagnostic factor in depression and anxiety. Behaviour Research and Therapy, 49(3), 186-193.

Mennin, D. S., \& Fresco, D. M. (2013). What, me worry and ruminate about DSM-5 and RDoC? The importance of targeting negative self-referential processing. Clinical Psychology: Science and Practice, 20(3), 258-267.

Moberly, N. J., \& Watkins, E. R. (2008). Ruminative self-focus and negative affect: an experience sampling study. Journal of Abnormal Psychology, 117(2), 314.

Moulds, M. L., Kandris, E., Starr, S., \& Wong, A. C. (2007). The relationship between rumination, avoidance and depression in a non-clinical sample. Behaviour Research and Therapy, 45(2), 251-261.

Mowrer, O. H. (1960). Learning theory and behavior. New York: John Wiley \& Sons, Inc.

Niles, A. N., Craske, M. G., Lieberman, M. D., \& Hur, C. (2015). Affect labeling enhances exposure effectiveness for public speaking anxiety. Behaviour Research and Therapy, 68, $27-36$

Nolen-Hoeksema, S. (1991). Responses to depression and their effects on the duration of depressive episodes. Journal of Abnormal Psychology, 100(4), 569.

Nolen-Hoeksema, S., Stice, E., Wade, E., \& Bohon, C. (2007). Reciprocal relations between rumination and bulimic, substance abuse, and depressive symptoms in female adolescents. Journal of Abnormal Psychology, 116(1), 198.

O’Toole, M. S., Jensen, M. B., Fentz, H. N., Zachariae, R., \& Hougaard, E. (2014). Emotion differentiation and emotion regulation in high and low socially anxious individuals: An experience-sampling study. Cognitive Therapy and Research, 38(4), 428-438. 
Olatunji, B. O., Naragon-Gainey, K., \& Wolitzky-Taylor, K. B. (2013). Specificity of rumination in anxiety and depression: A multimodal meta-analysis. Clinical Psychology: Science and Practice, 20(3), 225-257.

Plonsker, R., Gavish Biran, D., Zvielli, A., \& Bernstein, A. (2017). Cognitive fusion and emotion differentiation: does getting entangled with our thoughts dysregulate the generation, experience and regulation of emotion?. Cognition and Emotion, 31(6), 1286-1293.

Pond Jr, R. S., Kashdan, T. B., DeWall, C. N., Savostyanova, A., Lambert, N. M., \& Fincham, F. D. (2012). Emotion differentiation moderates aggressive tendencies in angry people: A daily diary analysis. Emotion, 12(2), 326.

Ponzetti Jr, J. J. (1990). Loneliness among college students. Family relations, 336-340.

Radloff, L. S. (1977). The CES-D scale: A self-report depression scale for research in the general population. Applied Psychological Measurement, 1(3), 385-401.

Russell, G., \& Shaw, S. (2009). A study to investigate the prevalence of social anxiety in a sample of higher education students in the United Kingdom. Journal of Mental Health, 18(3), 198-206.

Rusting, C. L., \& Nolen-Hoeksema, S. (1998). Regulating responses to anger: Effects of rumination and distraction on angry mood. Journal of Personality and Social Psychology, 74(3), 790.

Salkovskis, P. M. (1991). The importance of behaviour in the maintenance of anxiety and panic: A cognitive account. Behavioural and Cognitive Psychotherapy, 19(1), 6-19.

Schwarz, N., \& Clore, G. L. (2003). Mood as information: 20 years later. Psychological Inquiry, 14(3-4), 296-303. 
Selby, E. A., Anestis, M. D., \& Joiner, T. E. (2008). Understanding the relationship between emotional and behavioral dysregulation: Emotional cascades. Behaviour Research and Therapy, 46(5), 593-611.

Shields, M. C., Matt, L. M., \& Coifman, K. G. (2015). Physical activity and negative emotion during peer-rejection: Evidence for emotion context sensitivity. Journal of Health Psychology, 21(12), 2851-2862.

Suvak, M. K., Litz, B. T., Sloan, D. M., Zanarini, M. C., Barrett, L. F., \& Hofmann, S. G. (2011). Emotional granularity and borderline personality disorder. Journal of Abnormal Psychology, 120(2), 414-426.

Taylor, G. J., Bagby, R. M., \& Parker, J. D. A. (1997). Disorders of affect regulation: Alexithymia in medical and psychiatric illness. Cambridge, England: Cambridge University Press.

Thomsen, D. K. (2006). The association between rumination and negative affect: A review. Cognition and Emotion, 20(8), 1216-1235.

Treynor, W., Gonzalez, R., \& Nolen-Hoeksema, S. (2003). Rumination reconsidered: A psychometric analysis. Cognitive Therapy and Research, 27(3), 247-259.

Tugade, M. M., Fredrickson, B. L., \& Feldman Barrett, L. (2004). Psychological resilience and positive emotional granularity: Examining the benefits of positive emotions on coping and health. Journal of Personality, 72(6), 1161-1190.

Wong, Q. J., \& Moulds, M. L. (2009). Impact of rumination versus distraction on anxiety and maladaptive self-beliefs in socially anxious individuals. Behaviour Research and Therapy, 47(10), 861-867. 
Wong, Q. J., \& Moulds, M. L. (2011). Impact of anticipatory processing versus distraction on multiple indices of anxiety in socially anxious individuals. Behaviour Research and Therapy, 49(10), 700-706.

Zaki, L. F., Coifman, K. G., Rafaeli, E., Berenson, K. R., \& Downey, G. (2013). Emotion differentiation as a protective factor against nonsuicidal self-injury in borderline personality disorder. Behavior Therapy, 44(3), 529-540.

Zimmerman, M. (1994). Interview guide for evaluating DSM-IV psychiatric disorders and the mental status examination. East Greenwich, RI: Psych Products Press. 
Table 1.

Characteristics of Study 1 Participants $(n=54)$

\begin{tabular}{|c|c|c|c|c|c|}
\hline \multirow[t]{2}{*}{ Characteristic } & \multicolumn{2}{|c|}{$\begin{array}{l}\text { Clinical Sample } \\
(n=31)\end{array}$} & \multicolumn{2}{|c|}{$\begin{array}{l}\text { Healthy Controls } \\
(n=23)\end{array}$} & \multirow{4}{*}{$\begin{array}{l}\chi^{2}(1, n=54)= \\
2.67, p=.102\end{array}$} \\
\hline & $\mathrm{N}$ & $\%$ & $\mathrm{~N}$ & $\%$ & \\
\hline \multicolumn{5}{|l|}{ Sex } & \\
\hline Female & 21 & 68 & 20 & 87 & \\
\hline Male & 10 & 32 & 3 & 13 & \\
\hline \multicolumn{5}{|l|}{ Race/Ethnicity } & $\chi^{2}(2, n=54)=$ \\
\hline White/European & 27 & 87 & 21 & 91 & $.58, p=.749$ \\
\hline Black/African & 3 & 10 & 1 & 4 & \\
\hline Asian & 1 & 3 & 1 & 4 & \\
\hline \multicolumn{6}{|l|}{ Mental Health History } \\
\hline $\begin{array}{l}\text { Ever been on psychiatric } \\
\text { medication }\end{array}$ & 23 & 74 & 1 & 4 & $\begin{array}{l}\chi^{2}(1, n=53)= \\
25.19, p<.001\end{array}$ \\
\hline Ever been in therapy & 30 & 97 & 7 & 32 & $\begin{array}{l}\chi^{2}(1, n=53)= \\
25.72, p<.001\end{array}$ \\
\hline \multicolumn{6}{|l|}{ Current Employment Status } \\
\hline Employed full-time & 7 & 23 & 6 & 26 & $\begin{array}{l}\chi^{2}(1, n=54)= \\
.09, p=1.00\end{array}$ \\
\hline Employed part-time & 10 & 32 & 7 & 30 & $\begin{array}{l}\chi^{2}(1, n=54)= \\
.02, p=1.00\end{array}$ \\
\hline $\begin{array}{l}\text { Attending school or a } \\
\text { training program }\end{array}$ & 7 & 23 & 10 & 44 & $\begin{array}{l}\chi^{2}(1, n=54)= \\
2.67, p=.141\end{array}$ \\
\hline $\begin{array}{l}\text { Not employed due to } \\
\text { illness or disability }\end{array}$ & 4 & 13 & 0 & 0 & $\begin{array}{l}\chi^{2}(1, n=54)= \\
3.21, p=.127\end{array}$ \\
\hline
\end{tabular}

Note. The clinical sample consisted of $17(59 \%)$ individuals with generalized social phobia (GSP) with major depressive disorder (MDD) and 14 (41\%) individuals with GSP only. 
Table 2

Significant Two-way (Rumination by Negative Emotion Differentiation) Interaction in Predicting Social Avoidance in the Clinical Group $(n=29)$

\begin{tabular}{llcccccc}
\hline & $B$ & $S E$ & $95 \%$ CI & $s r^{2}$ & $R^{2}$ & $\Delta R^{2}$ \\
\hline Step 1 & Rumination & .00 & .06 & -.12 to .12 & .00 & $.28^{*}$ & \\
& Negative emotion & .21 & .27 & -.34 to .77 & .13 & & \\
& differentiation (NED) & & & & & & \\
& Mean negative affect & $.31^{* *}$ & .10 & .10 to .52 & .52 & & $.22^{* *}$ \\
Step 2 & Rumination & .04 & .05 & -.07 to .14 & .11 & $.50^{* *}$ \\
& NED & .16 & .23 & -.32 to .63 & .10 & & \\
& Mean negative affect & $.24 *$ & .09 & .05 to .42 & .39 & & \\
& Rumination $\times$ NED & $-.78^{* *}$ & .24 & -1.28 to -.28 & -.47 & & \\
& $F(4,24)=7.10, \mathrm{p}<$ & & & & & & \\
& .001 & & & & & \\
\hline
\end{tabular}

Note. $B=$ unstandardized coefficient; $S E=$ standard error; $\mathrm{CI}=$ confidence interval.

$* p<.05 ; * * p<.01$. 
Table 3.

Characteristics of Study 2 Participants $(n=193)$

\begin{tabular}{lcc}
\hline Characteristic & \multicolumn{2}{c}{ Study Sample } \\
\cline { 3 - 3 } Sex & $\mathrm{N}$ & $\%$ \\
Female & 153 & 79 \\
Male & 40 & 21 \\
Race & & \\
White/European & 157 & 81.3 \\
Black/African & 19 & 9.8 \\
Asian & 5 & 2.6 \\
American Indian/Alaska Native & 1 & 0.5 \\
Biracial/Multiracial & 9 & 4.7 \\
Other & 2 & 1.0 \\
Ethnicity & & \\
Non-Hispanic or Latino & 185 & $96 \%$ \\
Hispanic or Latino & 8 & $4 \%$ \\
Undergraduate Status & & \\
Freshman & 68 & 35.2 \\
Sophomore & 40 & 20.7 \\
Junior & 49 & 25.4 \\
Senior & 35 & 18.1 \\
Other & 1 & 0.5 \\
\hline
\end{tabular}


Table 4.

Significant Two-way (Rumination by Negative Emotion Differentiation) Interaction in Predicting Social Avoidance in the Sample $(n=190)$

\begin{tabular}{llcccccc}
\hline & $B$ & $S E$ & $95 \%$ CI & $s r^{2}$ & $R^{2}$ & $\Delta R^{2}$ \\
\hline Step 1 & Rumination & .03 & .01 & -.00 to .06 & .12 & $.14^{* *}$ & \\
& $\begin{array}{l}\text { Negative emotion } \\
\text { differentiation (NED) }\end{array}$ & $-.11^{* *}$ & .04 & -.19 to -.03 & -.19 & & \\
& Mean negative affect & $.05 *$ & .02 & .01 to .09 & .17 & & \\
Step 2 & Rumination & .02 & .02 & -.01 to .05 & .10 & $.18^{* *}$ & $.03 * *$ \\
& NED & $-.13^{* *}$ & .04 & -.21 to -.06 & -.23 & & \\
& Mean negative affect & .04 & .03 & -.01 to .10 & .15 & & \\
& Rumination $\times$ NED & $-.14 * *$ & .05 & -2.39 to -.03 & -.18 & & \\
& $F(4,185)=8.13, \mathrm{p}<$ & & & & & & \\
& .001 & & & & & & \\
\hline
\end{tabular}

Note. $B=$ unstandardized coefficient; $S E=$ standard error; $\mathrm{CI}=$ confidence interval.

$* p<.05 ; * * p<.01$. 


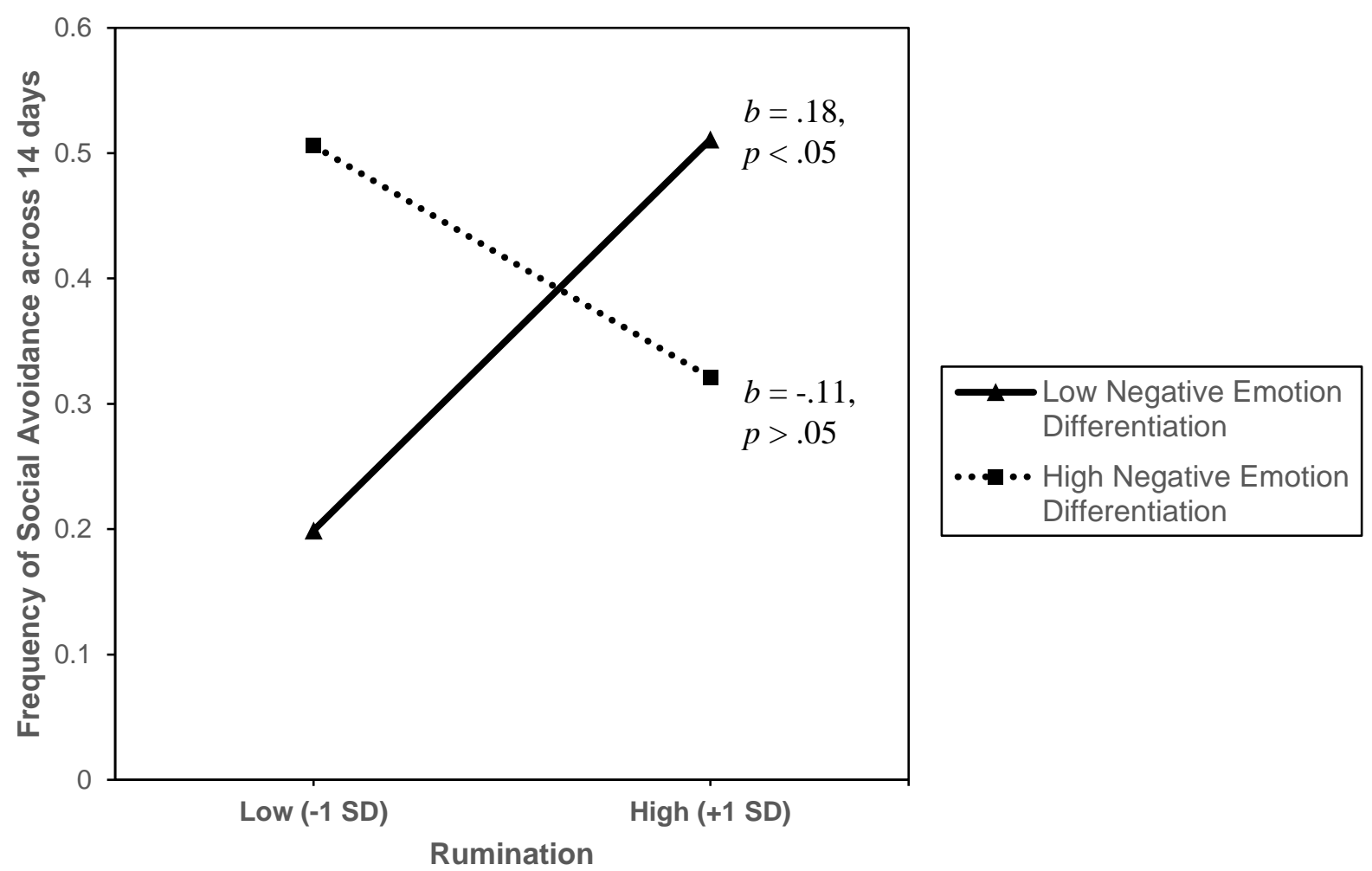

Figure 1. Study 1: This figure describes the interaction between rumination and negative emotion differentiation when predicting the frequency of social avoidance across the 14-day diary period in individuals with generalized social phobia with/without major depressive disorder. 


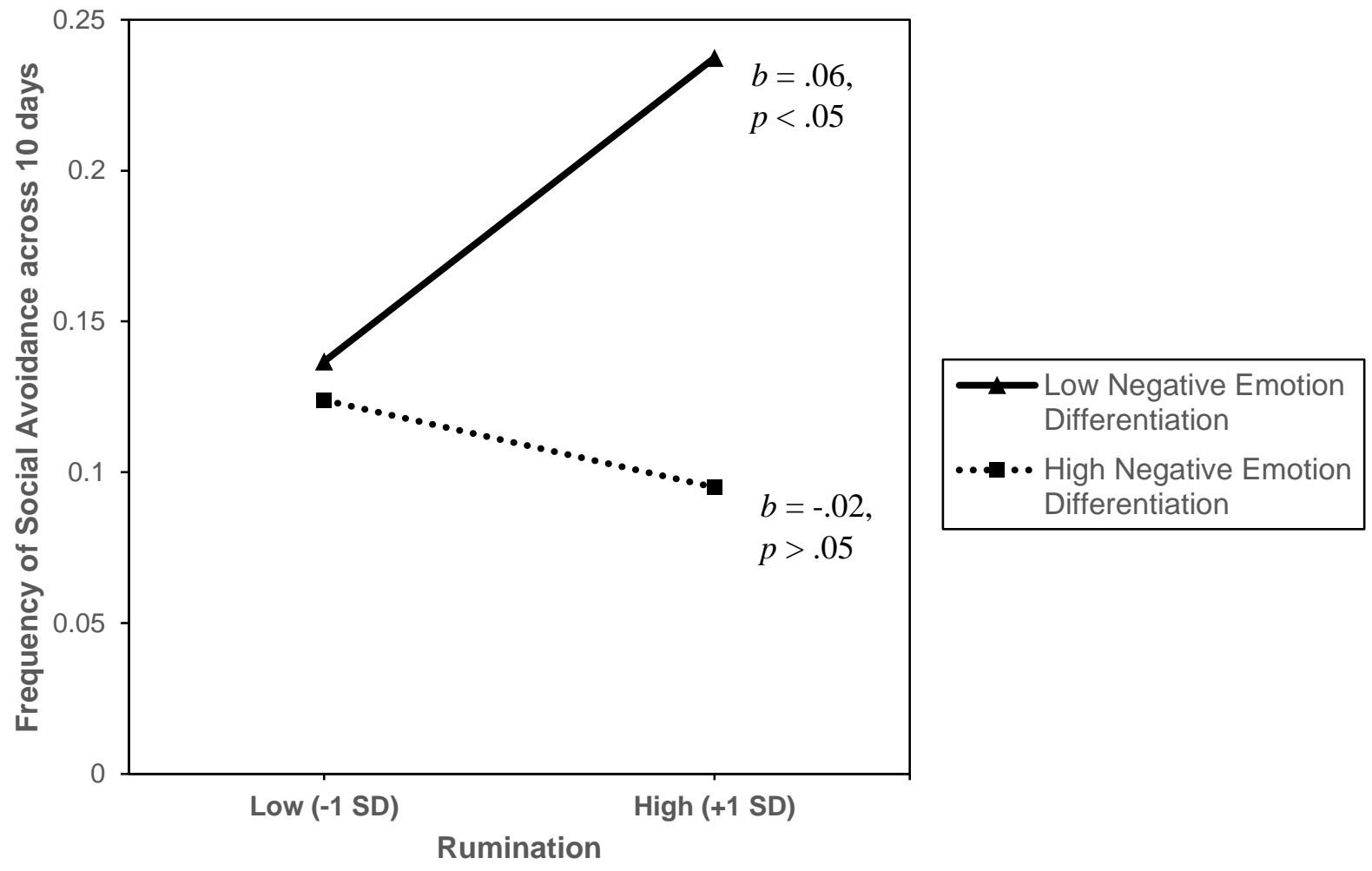

Figure 2. Study 2: This figure describes the interaction between rumination and negative emotion differentiation when predicting the frequency of social avoidance across the 10-day diary period in socially anxious college students. 